\title{
Adaptive Group Formation in Multirobot Systems
}

\author{
Ahmed Wagdy ${ }^{1}$ and Alaa Khamis ${ }^{2}$ \\ ${ }^{1}$ Robotics and Autonomous Systems (RAS) Research Group, German University in Cairo, New Cairo 12555, Egypt \\ ${ }^{2}$ Engineering Science Department, Suez University, Suez, Egypt
}

Correspondence should be addressed to Ahmed Wagdy; ahmedwagdyemam@gmail.com

Received 31 May 2013; Accepted 28 August 2013

Academic Editor: Thomas Mandl

Copyright (C) 2013 A. Wagdy and A. Khamis. This is an open access article distributed under the Creative Commons Attribution License, which permits unrestricted use, distribution, and reproduction in any medium, provided the original work is properly cited.

\begin{abstract}
Multirobot systems (MRSs) are capable of solving task complexity, increasing performance in terms of maximizing spatial/ temporal/radio coverage or minimizing mission completion time. They are also more reliable than single-robot systems as robustness is increased through redundancy. Many applications such as rescue, reconnaissance, and surveillance and communication relaying require the MRS to be able to self-organize the team members in a decentralized way. Group formation is one of the benchmark problems in MRS to study self-organization in these systems. This paper presents a hybrid approach to group formation problem in multi-robot systems. This approach combines the efficiency of the cellular automata as finite state machine, the interconnectivity of the virtual grid and its bonding technique, and last but not least the decentralization of the adaptive dynamic leadership.
\end{abstract}

\section{Introduction}

Any group of robots in a multirobot system (MRS) moving and coordinating together will always require the ability to quickly change group formation to adapt to the environment. All the robots within this system cooperate with each other to achieve the common goal of having the best group formation with decentralized communication between the robots in that system. This means that each robot has to consider the environmental changes, positions of other robots, and the global goal.

The multi-robot systems consist of either homogenous or heterogeneous robots. Homogenous robot system consists of a number of robots with the same properties, capabilities, configuration, and shape. On the other hand, heterogeneous robot system consists of robots that have different capabilities, properties, configuration, and shapes which makes task of implementing an algorithm to control their group formation without a centralized controller/coordinator difficult. Search and destroy, search and rescue, surround and conquer, and many military applications require multi-robot systems that are able to form a group and to adapt robustly.
In order to solve the group formation problem in MRS, it is required to

(i) model the relationship between robots in the same system,

(ii) avoid clashes between robots, obstacles and goal,

(iii) build all desired formations,

(iv) coordinate the motion of each robot,

(v) maintain formation while in motion,

(vi) develop an approach that ensures adaptability of the formation.

This paper presents a hybrid approach to group formation problem in multi-robot systems. This approach combines the efficiency of the cellular automata as finite state machine, the interconnectivity of the virtual grid and its bonding technique, and last but not least the decentralization of the adaptive dynamic leadership.

The remainder of this paper is organized as follows. Section 2 includes an introduction to multi-robot systems, their applications, and characterization. Some of the benchmark problems in multi-robot systems including the main 
topic of this paper will be covered in an attempt to identify the importance of group formation in multi-robot systems. Group formation problem is mentioned with details about its formulation and organizational paradigm.

Section 3 addresses the approaches that many researchers have developed to tackle the group formation problem. It starts by an introduction to the approaches that mimic the groups in nature, exploring the flocking behavioral-based approach and determining the flocking rules. The second approach introduced in that chapter is the virtual structure which included virtual springs and virtual grid; both techniques are used in describing the bonding relationship between the agents in the multi-robot system. The third approach introduced is the potential field and finally a new approach was introduced which is the dynamic leadership approach, and how this approach was developed and what part of the group formation problem it is solving.

Section 4 presents the proposed approach towards solving the group formation problem. This approach which is a hybrid approach mixing the well-established automata theory, virtual grid, and the newly proposed dynamic leadership algorithm to tackle the problem of the group formation is presented in this section.

Section 5 includes experiments on the proposed approach to investigate the validity of this approach in solving the group formation problem. Finally, concluding remarks and future work are summarized in Section 6.

\section{Group Formation in Multirobot Systems}

MRS is a group of robots that operate in the same environment and are designed with an aim of performing collective behavior. However, robotic systems may range from simple sensors, acquiring and processing data, to complex humanlike machines, able to interact with the environment in fairly complex ways. Some tasks may be quite complex for one robot to achieve or even impossible to be done, and when many robots try to achieve a goal it is high likely to be finished faster than if only one robot trying to accomplish that task and so the task completion time of a MRS would be minimal. Moreover, a MRS is more reliable than one robot, because, in a MRS, if one robot fails, there is always a substitute for that robot unlike the scenario for a single-robot system. The main advantage of a MRS is that it depends on "Power in numbers" which means that building several resource-bounded robots is easier and more economical than building a single powerful robot.

Group formation is observed in many animal species like flock of birds and school of fish, where members of a formation stay at a specific orientation and distance with respect to each other while moving, or fill a specific area as homogeneously as possible in an attempt to maintain a specific shape for their group. Group formation has permitted sophisticated behaviors that would have never been achievable by individual members. These behaviors include, but are not limited to, cooperative foraging, defense, search, or exploration. Most of the group formation techniques force the members of the group to move in a cohesive way as a whole.
2.1. MRS Aspects. Nearly all the work in cooperative mobile robotics began after the introduction of the new robotics paradigm of behavior-based control [1]. This behavior-based paradigm has had a strong influence in much cooperative mobile robotics research. Since the behavior-based paradigm for mobile robotics is rooted in biological inspirations, many cooperative robotics researchers have also found it instructive to examine the social characteristics of insects and animals and to apply these findings to the design of the MRS. Some of the main aspects of MRS are biological inspirations, communication, localization, mapping, and exploration, architectures, task planning, and control, object transport and manipulation, and last but not least motion coordination [2].

(1) Communication. The issue of communication in MRS has been studied extensively since the inception of distributed robotics research and has been divided into two paradigms: explicit and implicit communications [3].

(2) Localization, mapping, and exploration. An extensive amount of research has been carried out for single autonomous robots but only fairly recently has this been applied to MRS.

(3) Architectures, task planning, and control. The research area in this field addresses the issues of action selection, the delegation of authority and control, the communication structure, heterogeneity versus homogeneity in robots, achieving coherence in local actions, the resolution of conflicts, and other related issues.

(4) Object transport and manipulation. Enabling multiple robots to carry, push, or manipulate common objects cooperatively has been a longstanding, yet difficult, goal of any MRS. Many research projects have dealt with this topic area; few have been demonstrated on physical robot systems [4].

(5) Motion Coordination. Research themes in this domain that have been particularly well studied include path planning, traffic control, formation generation, and formation keeping. Most of these issues are now fairly well understood, although demonstrations of these techniques in physical multi-robot teams (rather than in simulation) have been limited [5].

2.2. Applications. The domain of traffic and transportation is geographically and functionally distributed to have a high degree of autonomy. Due to these very characteristics, many applications in this domain can be adequately modeled as a MRS. As a result of this modeling, it could be also possible to meet the growing interest in making traffic and transportation more efficient, resource saving, and ecological [6].

Moreover, applications of search and rescue requires large number of agents to solve the problem of the coverage area and so increase the efficiency of the system. Also, MRS can also be used in health care and medical curing 
such as eliminating cancer cells [7], management of organ transplants, ubiquitous access to medical information, and management of palliative patients.

Some effort has been put into the development of architectures that are optimized for space applications and include behavioral and cooperation patterns. The following is one of the space applications that involves MRS. Automatic Rendezvous and Docking, the automatic docking and rendezvous of spacecraft has been shown by a few space agencies. The first successful mission was by the Soviet Space Program in 1967. The satellite Kosmos-188 (SIZE-ALONE) achieved the world's first automatic docking with the artificial Earth satellite Kosmos-186 [8].

2.3. Group Formation Challenges. There are many generic problems facing group formation in any MRS; the following is some of these problems.

(1) Modeling of the relationship between robots in the same system: a mathematical or physical connection that relate all the members with each other.

(2) Avoiding clashes between robots, obstacles, and goal: this problem depends not only on how agents are organized in a formation, but also it depends on the sensors (hardware) installed on the members.

(3) Coordination of the motion of each robot: it includes formation control and coordination between the members of a system in order to maintain the formation while in motion.

(4) Determining the reaction of other robots due to change in motion: ability to predict changes through learning.

(5) Finding the best path: determining the shortest and most efficient path regarding the consumption of energy.

\section{Related Work}

There are many algorithms mimicking the group formations in nature such as birds flocking, fish schooling, and quadrupeds herding. These groups are considered effective if members attain performance goals regarding quality, quantity, and timeliness of work results. Generally, maintaining a formation is accomplished in two steps: a perceptual process, detect-formation-position, determines the robots proper position in formation based on current environmental data; the second step is the motor process maintain formation where commands are sent to motors to direct the robot toward the correct location.

3.1. Flocking. Behavior-based approach desires to prescribe specific behavioral characteristics to each robot, but it is difficult to analyze the approach by theoretical formalization and so it is not easy to guarantee the convergence of the robots into the desired formation. Flocking is a coordinate and cooperative behavior easily conspicuous in a large number of beings, ranging from simple bacteria to mammals. This

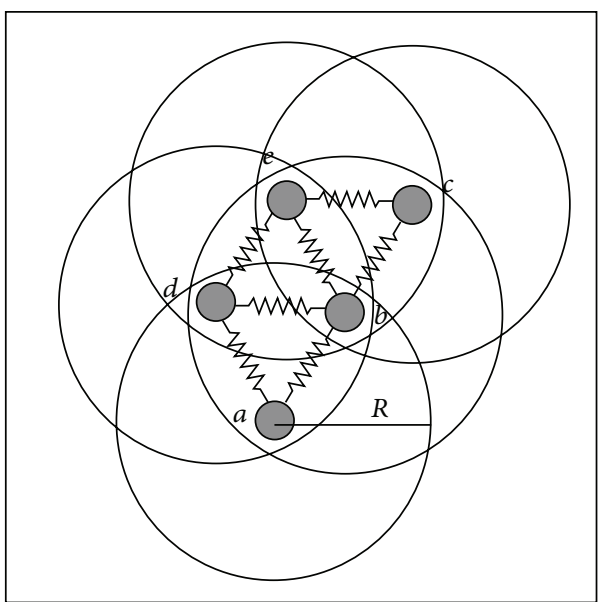

FIGURE 1: Virtual spring between agents.

salient behavior is predominantly based on the principle that there are safety and strength in numbers [9]. Bird flocking has been studied for many decades and many algorithms were suggested to mimic this behavior at which flocking is considered as free flocking or constrained flocking. Free flocking is referred to the behavior of flocking in an environment that does not have any obstacles and so the members of this group move freely, while in constrained flocking the environment will have obstacles and it might have many other groups flocking in the same environment which could be modeled as moving obstacles. The engineering applications of flocking include massive mobile sensing in an environment and several military missions like attack tactics [10-12]. Reynolds introduced three heuristic rules that led to the creation of the first computer animation of flocking. These three rules are also known as cohesion (or flock centering), separation (or obstacle avoidance), and alignment (or velocity matching) [13].

3.2. Virtual Structure. The virtual structure approach which models each robot as a particle in a single structure and the control of robot formation is straight forward, but this approach requires huge communication bandwidth between members of the formation.

(1) Virtual Spring. Fujibayashi et al. [14] proposed a probabilistic control technique which makes use of virtual springs to keep two robots in close proximity; this technique uses only local information and it is decentralized. Each pair of robots is connected to each other via a virtual spring illustrated in Figure 1. These virtual springs are created between members of a formation probabilistically; the candidate springs to be created are chosen depending on the number of connections the robots it joins have. It is difficult to choose custom parameters for each formation due to the randomness of the algorithm in choosing the created or the broken springs. However, Daneshvar and Shih [15] used a virtual spring-based model as a model for group coordination of autonomous flying agents to maintain safe distance between the members of the formation. Their proposed approach 


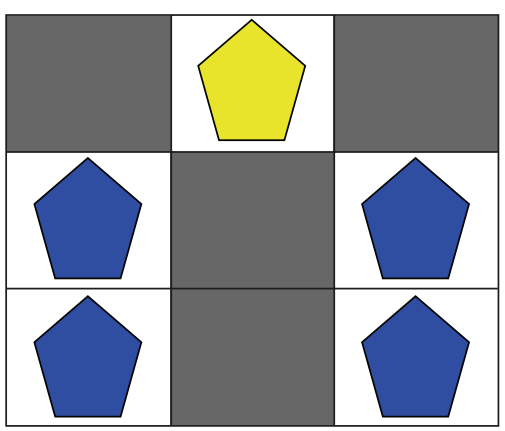

FIGURE 2: Small virtual grid.

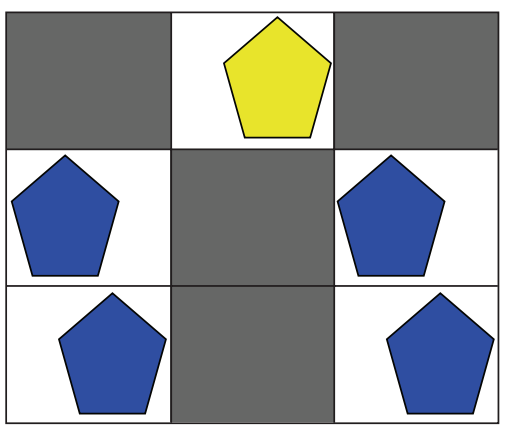

FIGURE 3: Large virtual grid.

forms a virtual connection between a vehicle; and its neighbor vehicle, as the vehicle changes its position, speed and altitude the total resultant forces on each virtual spring try to maintain zero by moving to the mechanical equilibrium point.

(2) Virtual Grid. Virtual grid is a new bonding technique introduced here that describes the bond between members in a formation at which virtual cells bound the whole formation. The number of cells in a grid corresponds to the number of robots in the formation and this means that each robot may only move within only one cell. The whole grid moves in the direction of the goal position while maintaining the formation of the group. The length and width of each cell determine the tolerance and error of a robot position in a formation. In other words, if it is required to have very strict formation shape, the length and the width of the cells should be as small as possible to avoid large deflections from the desired formation shape as illustrated in Figures 2 and 3. Despite the fact that decreasing the length and the width of the cells will improve the formation error, it will also increase the computational time and will cause the system to be stiffer and therefore will take longer time to reach its final position.

3.3. Potential Field. Cai and Yang [16] have proposed the potential field particle swarm optimization technique with a goal of finding all potential target points in unknown environment by assigning alike charges to the robots and obstacles and assigning opposite charges to targets and collision-free pathway points then combining the particle swarm optimization technique to find the optimal or near optimal collision-free pathway points. This algorithm has
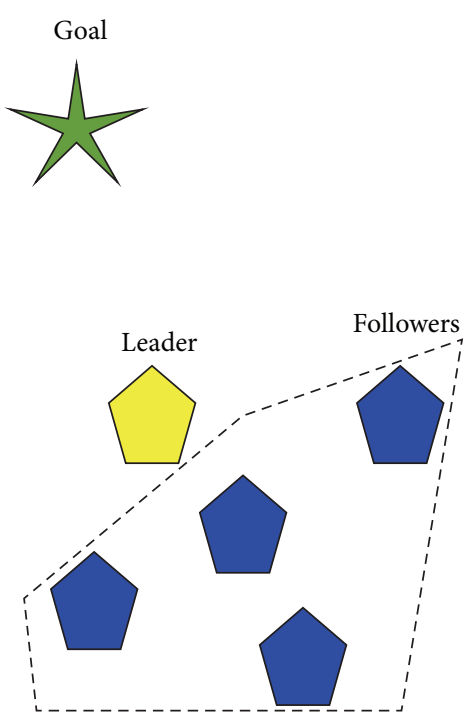

Figure 4: Choosing a leader.

been applied to known and unknown environments; for unknown environments the way points are always in change due to the situations of newly discovered areas. The potential field is an approach commonly used to explore unknown environments where robots are supposed to flexibly interact with dynamic environments.

3.4. Dynamic Leadership. Introducing a new technique to effectively change the centralized approaches into decentralized ones, the dynamic leadership rules are to be embedded within each robot in a formation to ensure that all robots will abide by the same rules. Simply, these rules are just standards that identify the leader of the formation for an undefined time only bounded by the fitness of the current leader. A robot is considered fit to be a leader of a formation only if this robot is superior to all other robots in the formation regarding the leadership rules, for example, closeness to goal or better connection to large number of members within the formation. These rules force the change of the leader frequently according to the fittest robots position. As seen in Figure 4, the agent indicated in yellow color would be eligible for leadership as it is the closest to the goal point and also has the widest communication circle.

\section{Proposed Approach}

A hybrid approach mixing the well-established automata theory, virtual grid, and the newly proposed dynamic leadership algorithm to tackle the problem of the group formation is presented in this section.

4.1. Automata Theory. Automata is the plural of automaton, and it means "something that works automatically." An automaton is a pointed, observable representation of an algebraic structure [17]. The elements of this structure are the inputs to the automaton and the elements of the representation are the states of the automaton. Pointed refers to the fact 
Input: Agents in a system

Output: Assigned Leader of the system

(1) Assign the Leadership to any random agent within the system;

(2) for $n$ thCounter $\leftarrow 1$ to $n_{A}$ do

(3) Assign a candidate leader to the $n$ thCounter agent in the system;

(4) Compute the ObjectiveFunction of the candidate leader;

(5) if current leader ObjectiveFunction < candidate leader ObjectiveFunction then

(6) Assign the candidate leader to be the current leader;

Algorithm 1: Assigning leadership.

that the representation has a distinguished element, which is treated as the start state of the automaton. Observable means that there is a function from the representation to some (possibly structured) set, for example, the set accept, reject. The concepts of automata theory have important use in designing and analyzing computational models of several hardware and software applications. These concepts are abstract in nature and hence can be used to model many challenging systems.

4.2. Virtual Grid-Based Interconnection Structure. As mentioned in the previous section, virtual grid can be used as an internal bonding structure that relates agents in a system to each other and so build a virtual connection between the agents. The new bounding relation that is created by the virtual grid acts as a cohesive force. The smaller the cells in the grid are, the stronger the cohesive force is. However, this strength comes with a cost of longer computational time and unstable control of the system due to the small allowed error tolerance. Therefore, it is required to assess the importance of having a stiffer bond over an unstable system, and this assessment could take into consideration the task that this formation will be executing.

4.3. Dynamic Leadership. This algorithm rules are to be embedded within each robot in a formation to ensure that all robots will abide by the same rules. Simply, these rules are just standards that identify the leader of the formation for an undefined time only bounded by the fitness of the current leader. A robot is considered fit to be a leader of a formation only if this robot is superior to all other robots in the formation regarding the leadership rules.

(1) Objective Function. In an attempt to develop rules that characterize the properties of a leader in a MRS, the following objective criterion was developed:

$$
\text { Objective Function }=\frac{w_{1}}{\sum_{i=1}^{n_{C}} \mathrm{DAC}_{i}}+\frac{1-w_{1}}{D},
$$

where

(i) $w_{1}$ is weight for communication radius; (ii) $D$ is distance between agent and goal;

(iii) $n_{C}$ is number of corners in the formation;

(iv) DAC is distance from agent to corner.

Other objective functions such as maximizing the battery life or minimizing the power consumed might be also developed by decreasing the time used for communication between robots in the MRS.

If the environment is unknown, therefore there is no target location known to the system and so the parameter responsible for the weight given to the closeness of the target (goal) would be 0; Algorithm 1 shows how (1) is used in assigning a leader to the MRS.

The proposed approach to tackling the problem of the group formation is a hybrid approach mixing the wellestablished automata theory, virtual grid, and the newly proposed dynamic leadership. However, the automata theory has a disadvantage of having rigid states, that is, an agent is not considered in a state unless it has reached a specific position while not taking into consideration that there are always practical errors and divergence when it comes to agents' motion. This rigidity is considered as disadvantages to the automata theory if used in this approach. Also, the virtual grid technique has a disadvantage of either it allows a tolerance in the displacement between agents that might cause large divergence from the desired positions or an unstable control system if the grid cells were too small and does not allow any tolerance.

On the other hand, the automata theory has an advantage of ensuring that the desired goal is achieved in form of milestones (checkpoints) and so provide the system with prompt feedback about the formation status, while the virtual grid provides the agents in the system with an interconnection technique that bonds the agents together and controls their behavioral activity. In this hybrid approach the rigidity of the automata theory is balanced by combining the allowance (tolerance) of the virtual grid technique with it, that is, allowing an agent to be considered in a state if its position is within the cell of the virtual grid. Therefore, this hybrid approach targets the efficiency and the mathematical model of the automata theory, the interconnectivity of the virtual 


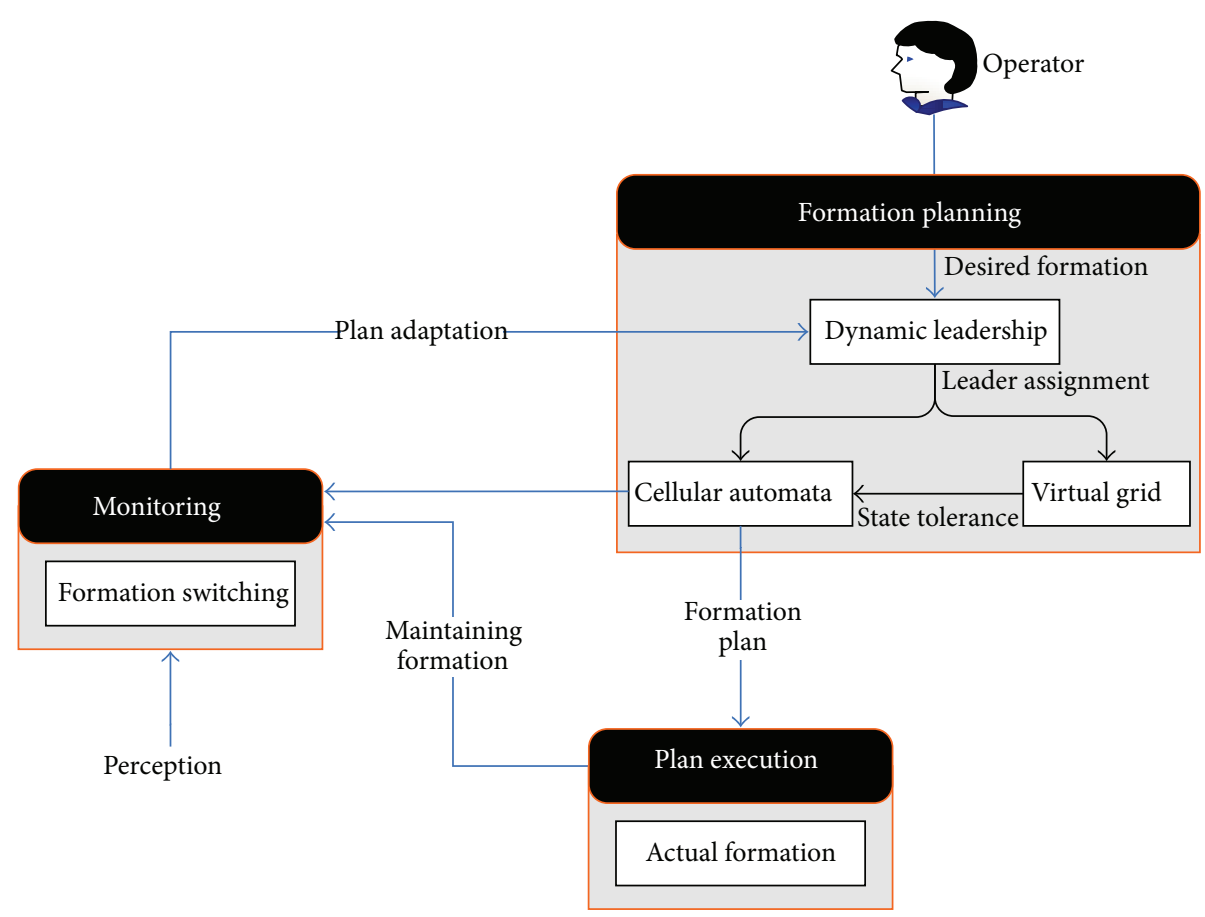

FIgURE 5: Hybrid approach.
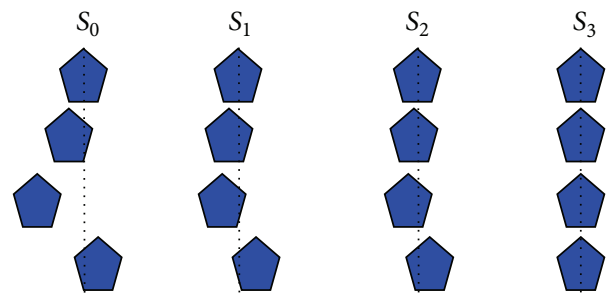

FIGURE 6: Discretized states.

grid and its bonding technique, and last but not least the decentralization of the adaptive dynamic leadership. Basically, this approach is a sum of the advantages and suppressant of the disadvantages of its ingredients. Figure 5 shows how the proposed approach being used in the Formation PlanExecute-Monitor loop.

(2) Formation Planning. Automata theory is used to store the discretized scene states where these states are defined as virtual steps or checkpoints that assure that all agents will reach the final formation shape at the same time and so will ensure that communication between agents is intact and that no agent dropped out of the formation. Linear discretization of the scenes would be computed with respect to the number of agents in a system. This means that for a system of 4 agents it is expected to have 4 states (Figure 6), $S_{0}$ is starting state, $S_{1}$ is first intermediate checkpoint, $S_{2}$ is second intermediate checkpoint, and $S_{3}$ is end positions and the final formation shape

$$
n_{S}=\operatorname{ciel}\left(\frac{n_{A}-n_{C}}{n_{L}}\right)
$$

where

(i) $n_{L}$ is number of lines in a closed shape, where $n_{L}>0$,

(ii) $n_{C}$ is number of corners in a shape;

(iii) $n_{A}$ is number of agents in a formation;

(iv) $n_{S}$ is number of centers on a line;

(v) $d_{C}$ is distance between corners;

(vi) $d_{A}$ is diameter of an agent.

If $n_{S}>1$, then the distance between the corners must be increased to make sure that a line in the formation will hold more than two agents.

Figure 7 shows how 5 agents would be organized in a rectangular formation by increasing the number of agents that a line can hold. In the case of 5 agents a center would be added to all the virtual lines and the corner to corner distance would be computed using the following:

$$
d_{\text {Cnew }}=\left(d_{\text {Cold }} \times n_{S}\right)+d_{A} .
$$

(3) Formation Execution. Algorithm 2 is implemented in each robot in the MRS to determine its position in the desired formation and ensures that all robots are equidistant from each other. However, this algorithm only arranges the agents in line formation.

Figure 8 shows a 7-agent multi-robot system applying a rectangular formation and using the previously mentioned equations to compute the positions of the agents in the 
Input: Agent and Desired Formation

Output: End position

(1) if Agent $==$ Leader then

(2) End position = Current position;

(3) Assigned position = Current position;

(4) for corner $\leftarrow 1$ to $n_{L}$ do

(5) for $n$ thCounter $\leftarrow 2$ to $n_{A}$ do

(6) Agent AthCounter $_{\text {End position } \rightarrow \text { Assigned }}$ position $+\left(d_{A} \times n\right.$ thCounter $)$

(7) Assigned position $=$ Assigned position $+d_{C}$ else

(8) Get End position from Leader.

Algorithm 2: Position assignment (Desired formation).

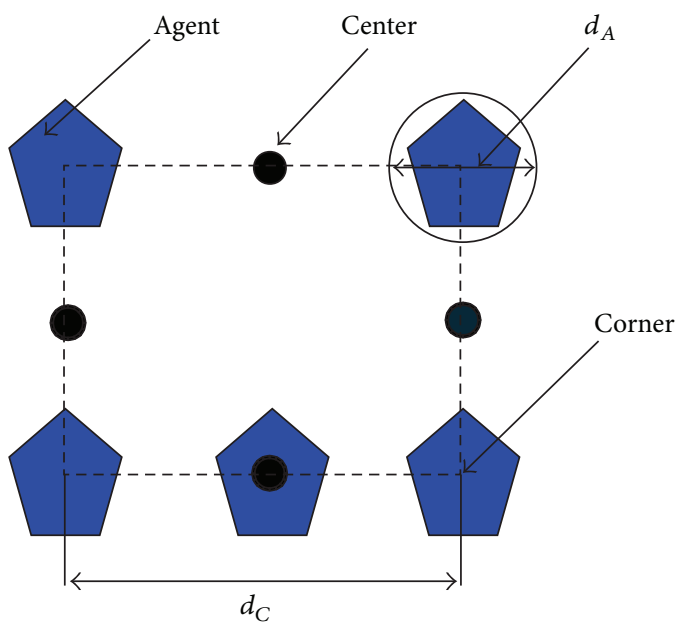

Figure 7: 5 Agents rectangular formation.
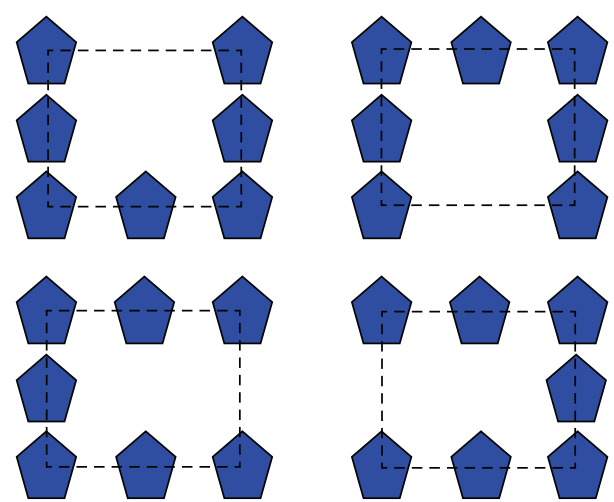

FIGURE 8: Seven agents rectangular formation.

formation. There are four possible combinations to reach the desired formation.

(4) Pathway Discretization. The distance between the start point and the desired end point of a robot is called the pathway. This pathway is discretized using linear method into a number of points according to the number of agents (robots) in the system. However, this does not ensure the maintenance of the formation while moving to the goal, so here again
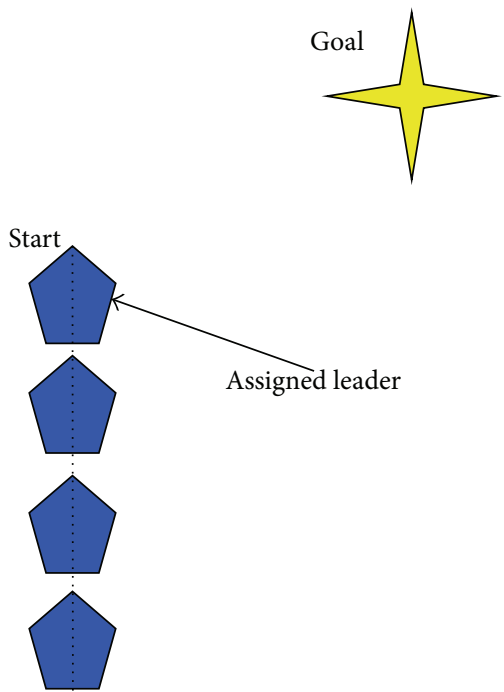

Figure 9: Motion to goal scenario.

automata theory is being used to divide the pathway points unequally but synchronous with the checkpoints of all the members of the formation. The leader determines its shortest path to the goal and then broadcasts the next checkpoint of each member to them. Figure 9 shows a scenario where it is desired to move the formation from the starting point to the goal.

The automata theory provided the hybrid approach with a method to continuously have feedback whether the desired action was performed or not. This method is the states (checkpoints) at which the agent broadcasts its current reached state to the whole MRS and so it can be determined if the desired goal was reached; if the goal is not reached, then the agent that did not reach the checkpoint continues to alter its position. The following steps are executed to reach the final goal position.

\section{Step 1. Assign a leader using Algorithm 1.}

Step 2. Assign a goal position to each agent, shown in Figure 10, using Algorithm 2. 
Input: Agent, Current Formation, and map

Output: Agents' new position

(1) call Assigning leader as in Algorithm 1;

(2) call Position Assignment \{Line Formation\} as in Algorithm 2;

(3) while obstacle not passed do

(4) Agents continue moving;

(5) call Position Assignment \{Current Formation\} as in Algorithm 2;

Algorithm 3: Adaptive formation switching.

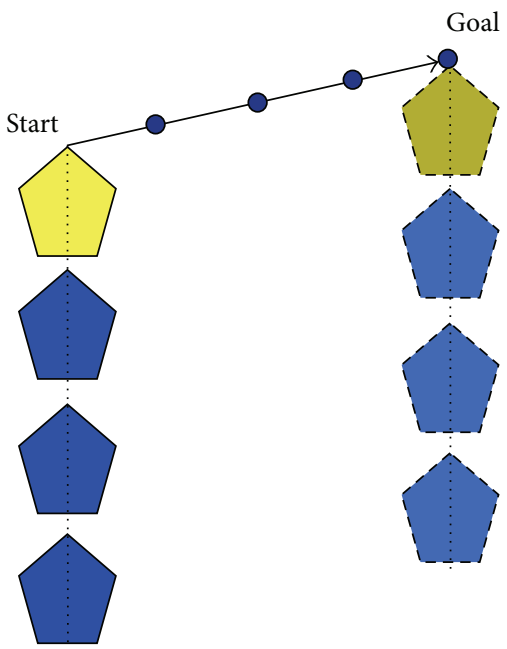

Figure 10: Pathway.

Step 3. Each agent discretizes its pathway into foreseen checkpoints (Figure 11).

Step 4. Each agent moves towards its checkpoints as shown in Figures 12, 13, and 14, and when it reaches the checkpoint it acknowledges the whole MRS and awaits until all the agents in the system reach the same state (checkpoint).

Step 5. Repeat Step 4 until reaching the goal position for all agents (Figure 15).

(5) Formation Switching. Switching between different formations is the ability of the MRS to identify its current formation and identify its surroundings in the environment then choose another formation to switch to. Algorithm 3 is used to adaptively pass around an obstacle or even pass in a narrow passage then return back to the initial formation.

\section{Experimental Results}

The testing was set up using a free software used for simulation called Virtual Robotics Experimental Platform (VREP) [18]. This simulation program is developed by Coppelia Robotics and it include all the modules of the Khepera III robot that was planned to be used practically to test the approach.

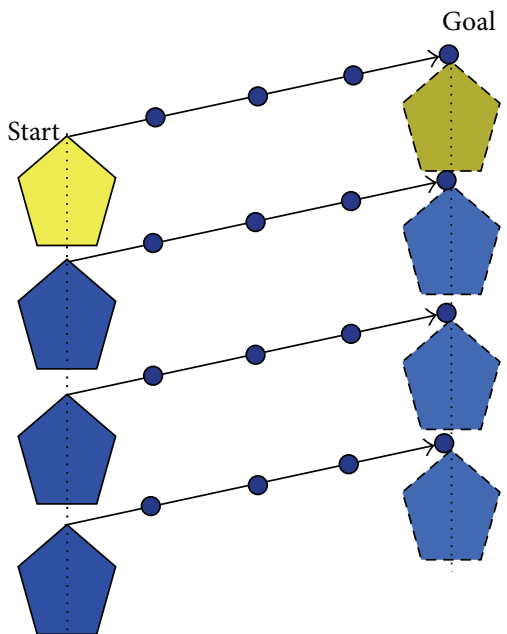

FIgURE 11: Broadcast.

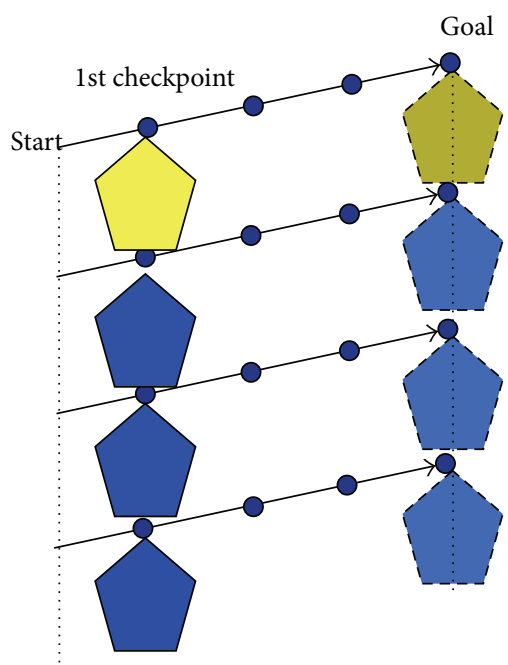

FIgURE 12: Scene 1.

Setting up an experiment requires understanding both the practical and the simulation models and identifying the communication method between agents in the MRS. In all the experiments conducted, it is assumed that the leader is the initiator of the formation, and these agents building a formation will be used to build all different formations to 


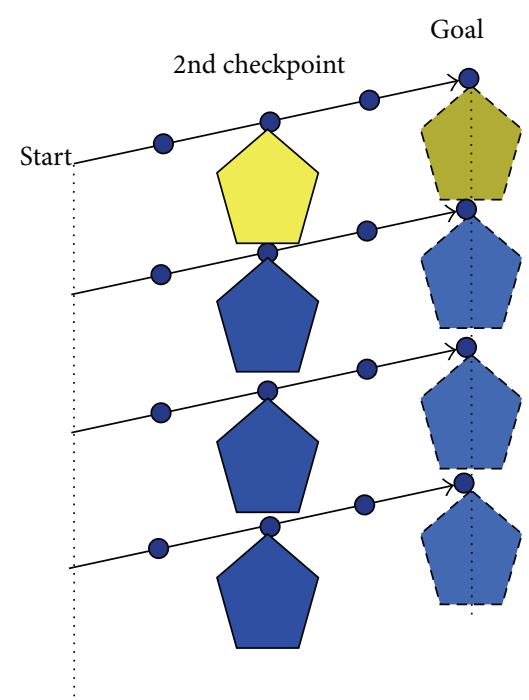

FIGURE 13: Scene 2.

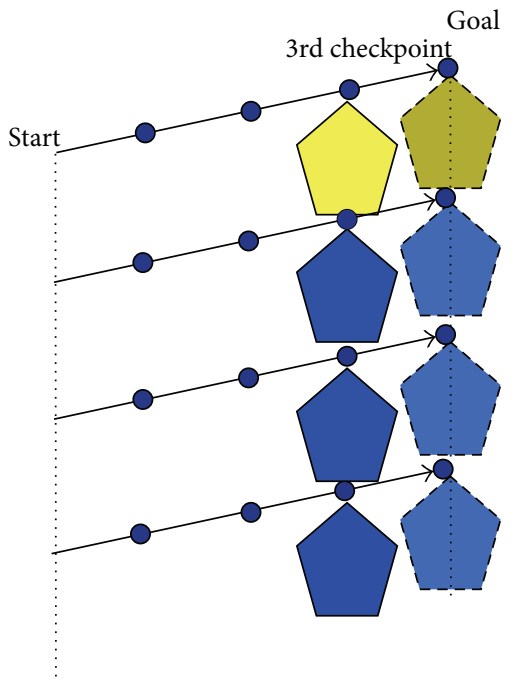

FIGURE 14: Scene 3.

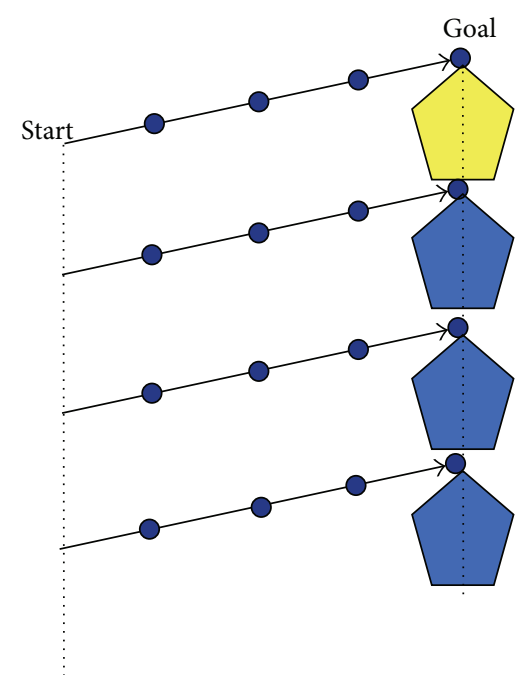

Figure 15: Final goal.

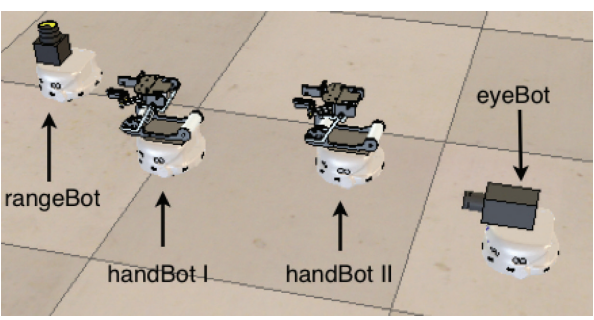

FIGURE 16: Four agents experiment setup.

maintain consistency in the results. All formations will have an equal chance to perform well and achieve the goals of the collaborative activity, although this may conflict with the best interest of individual agents. Therefore, to form the groups, the leader has to evaluate the collaboration goal in a way that satisfies both the task of the collaboration that the agents have to achieve as a group and the individual needs of the agent. However, the interest in collaboration in the group formation will be only regarding the process of building up a formation and maintaining this formation throughout the motion of the whole group in the environment.

A simulation environment of four agents, Figure 16, is used in testing the proposed algorithm as most of the formations would require at least 4 agents to build them. However, some basic formations such as line formation have no limit regarding the number of agents used to build it. Each one of the agents in the MRS will have a distinct capability; that is, Agent 1 will have a laser finder module (rangeBot), Agents 2 and 3 would have a gripper module (handBot), while Agent 4 will have a camera module (eyeBot). The workstation used for running the simulations had a $2.26 \mathrm{GHz}$ Intel core 2 Duo processor with a cache memory of $3 \mathrm{MB}$.

5.1. Evaluation Metrics. In these experiments, the point of interest is the evaluation of the group formation in terms of how well the formation was built rather than how well the formation will perform; therefore, the collaboration goals for the agents and the formation are modeled as a set of requirements (constraints) and so the success of group formation in this context is defined by the satisfaction of the constraints that define these goals. To achieve this, the following assumptions were set.

(i) All agents within the MRS have equal communication radius.

(ii) Each agent in the environment should belong to the group (i.e., no agents are delinked).

(iii) All agents in the MRS should be used in the building of any formation, and all built formations are stable.

(iv) Each agent is able to identify all the members of the MRS.

(v) Each agent is able to distinguish between an agent and an obstacle.

(vi) All agents have the same behavioral wonder action. 


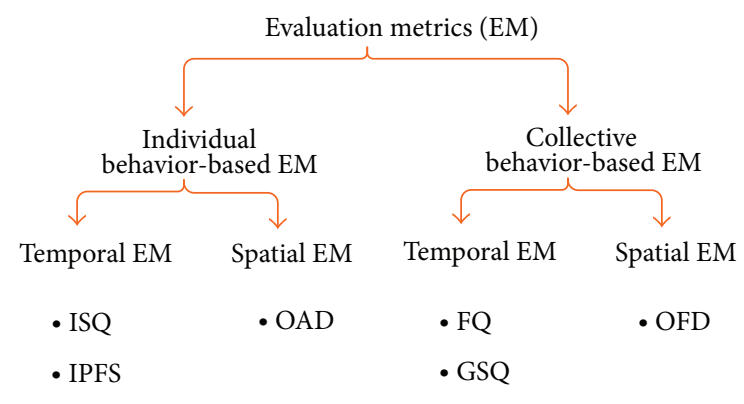

FIGURE 17: Evaluation metrics.

(vii) The error tolerance accepted would be a maximum of $15 \%$ determined by the error in the proximity sensor [19].

The Evaluation Metrics are classified into two categories: Individual-based EM and Collective-based EM as illustrated in Figure 17. A further classification would be position-based and time-based EM.

Constraints are defined as any parameter, variable, or condition that affects the process of the group formation, that is, environment structure and position of the goal. The following is a descriptive definition of the EM used.

5.1.1. Individual Behavioral EM. (1) Individual satisfaction quality (ISQ) is used to determine how well the intermediate states were satisfied in the formation of the group (allocation of agents). ISQ is classified as one of the time-based EM and is quantitatively measured by the average time taken (ATT) by each agent to reach intermediate states and finally the goal position using the following:

$$
\mathrm{ISQ}=\sum_{i=1}^{s} \frac{T_{i}}{s},
$$

where

(i) $s$ is the number of intermediate states,

(ii) $T$ is the time taken by an agent to reach a state.

(2) Individual perceived formation satisfaction (IPFS) is used to refer to how pleased is the individual with being allocated at the assigned position in the formation. This metric is based on the different environmental obstacles and power needed to reach the final position. Basically, IPFS is considered as a time-based evaluation metric and it is concerned with the energy exerted by the agent in the still state while trying to reach the goal position. IPFS is computed as the battery consumption duration during still states which is determined by the following:

$$
\mathrm{IPFS}=T_{t}-\frac{D_{\text {theoretic }}}{V},
$$

where

(i) $T_{t}$ is the total time taken by an agent to reach all the intermediate states and the goal state;

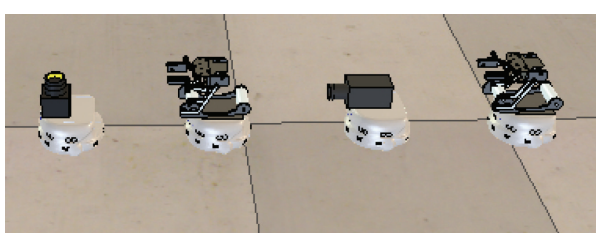

FIGURE 18: Line formation.

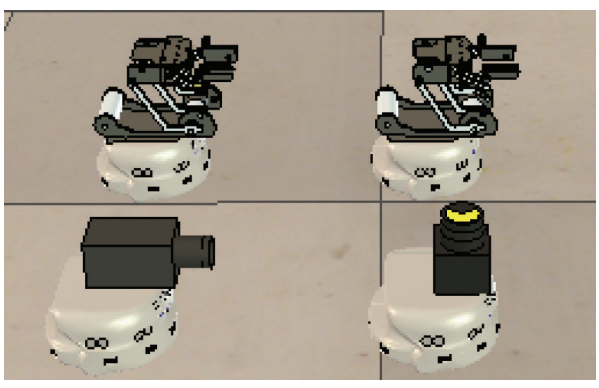

FIgURE 19: Rectangle formation.

(ii) $V$ is the velocity of the agent (constant in all the experiments at $64.4 \mathrm{mms}^{-1}$ );

(iii) $D_{\text {theoretic }}$ is the theoretical distance that the agent will cover to reach the goal.

(3) Optimal Agent Divergence (OAD) is a metric used to determine the divergence of the agent actual state from the desired one and it sets the acceptability of this reached state with respect to a predefined tolerance which is defined by the task that the whole MRS will perform. OAD is classified as a position based evaluation metric and is calculated using the following:

$$
\mathrm{OAD}=\left\|P_{\mathrm{Ds}}-P_{\mathrm{As}}\right\|,
$$

where

(i) $P_{\mathrm{Ds}}$ is the desired state position;

(ii) $P_{\mathrm{As}}$ is the actual reached state position.

5.1.2. Collective Behavioral EM. (1) Formation quality (FQ) identifies how well all the agents within the group collaborated together in order to build a formation and how long it took them all to reach the desired formation. FQ is a timebased evaluation metric that is calculated using (7) which is the ATT for all agents to reach the final state and so reach the desired formation structure and then compared to the Optimal Building Time as follows:

$$
\mathrm{FQ}=\sum_{i=1}^{n} \frac{T_{F_{i}}}{n}
$$

$$
\text { Optimal Building Time }=\sum_{i=1}^{n} \frac{V}{D_{\text {theoretic }_{n}}} \text {, }
$$

where

(i) $n$ is the number of agents in the MRS; 


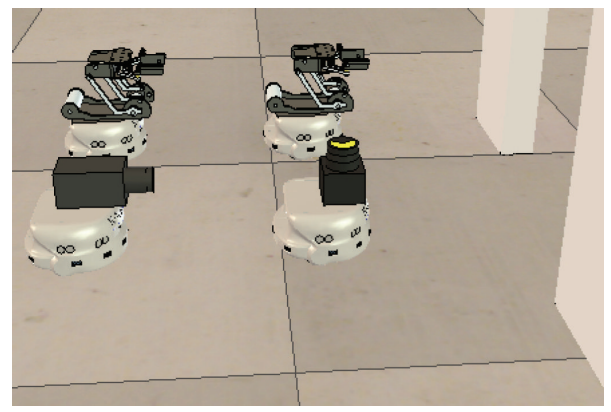

Figure 20: Rectangle formation facing narrow passage.

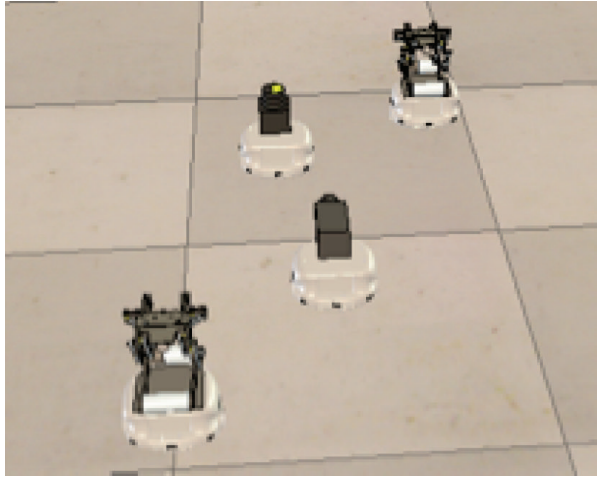

(a) Initial position

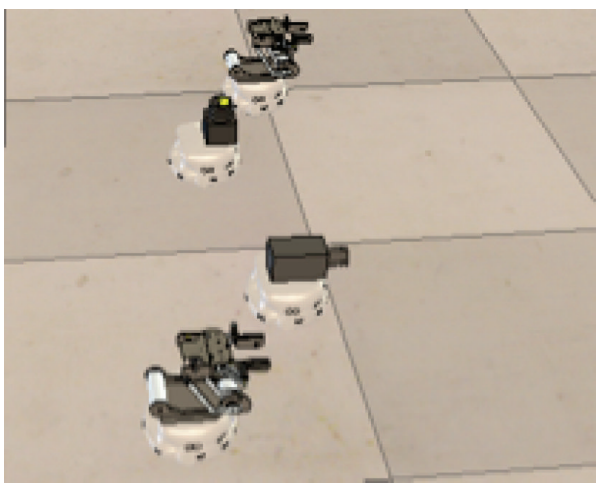

(c) Motion in $x$

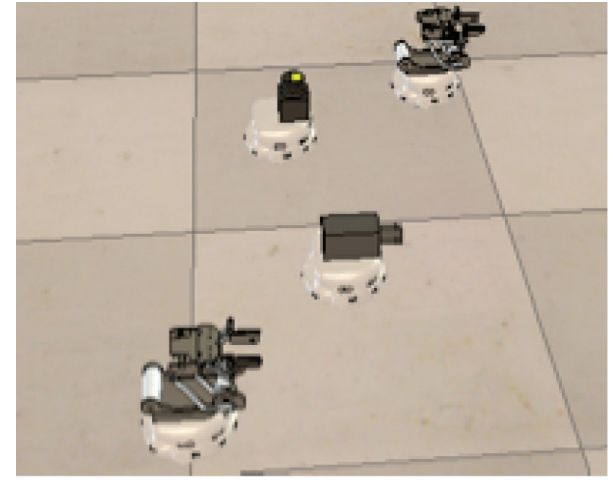

(b) Orienting

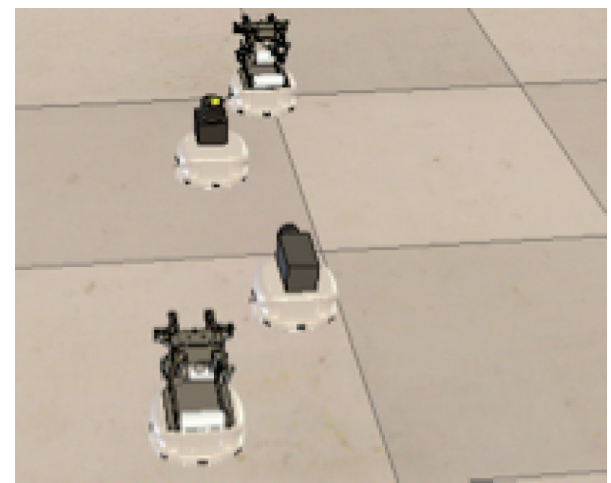

(d) State 1 achieved

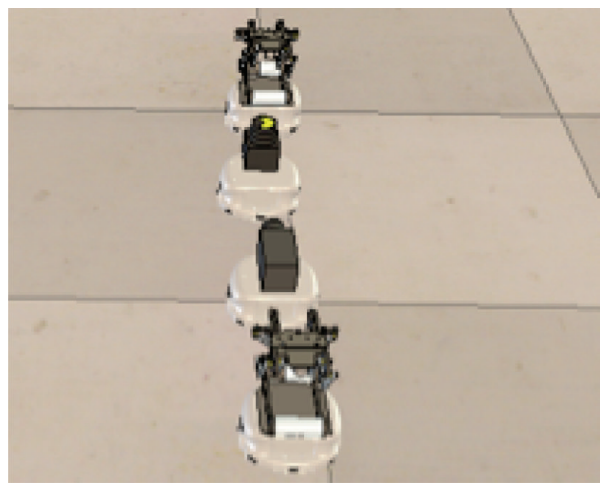

(e) Final position

FIGURE 21: Line formation scenario. 


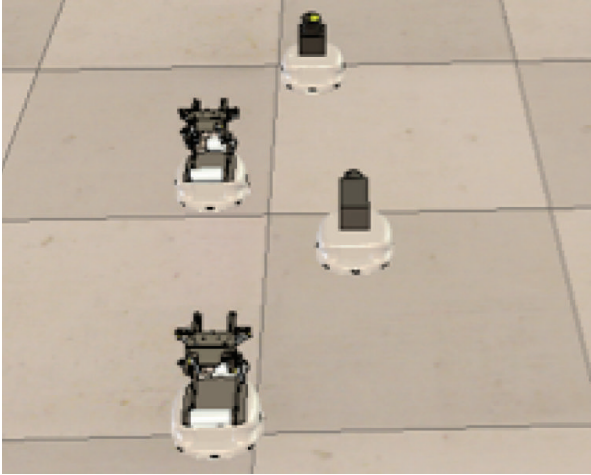

(a) Initial position

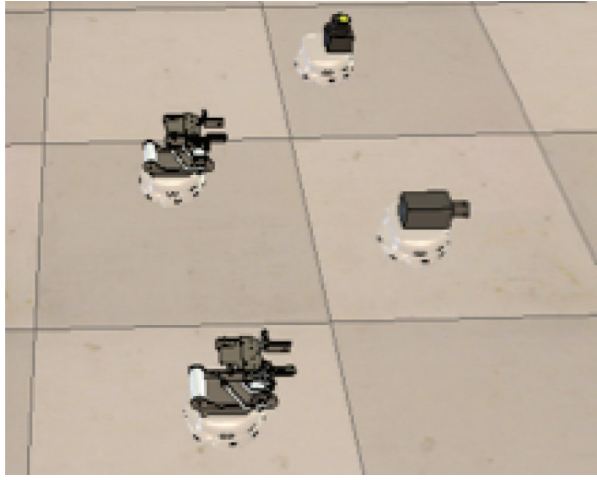

(c) Motion in $x$

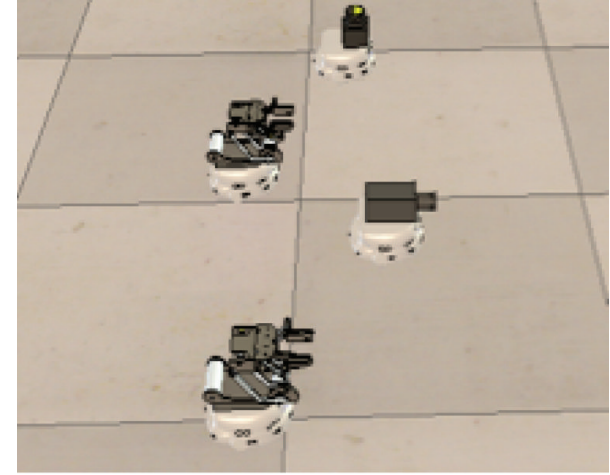

(b) Orienting

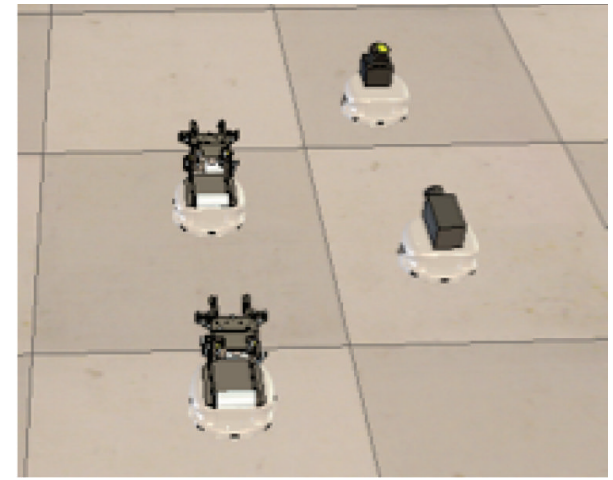

(d) State 1 achieved

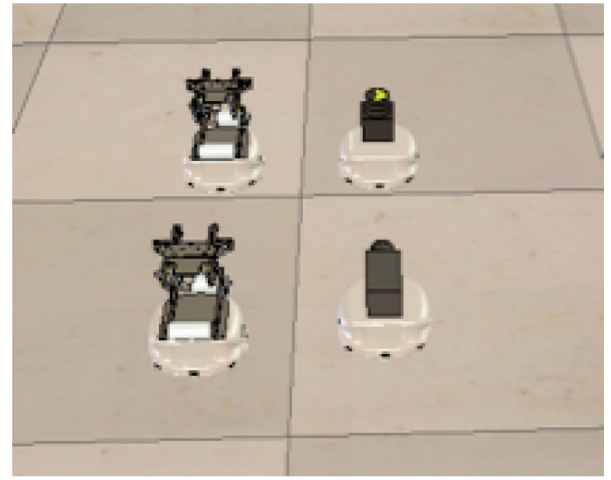

(e) Final position

FIGURE 22: Rectangle formation scenario.

(ii) $T_{F}$ is the time taken by an agent to reach the final position.

(2) Goal satisfaction quality (GSQ) is used to identify how well the MRS reached the goal position; this includes the different formations that were applied on the system in order to reach this goal position. GSQ is a time-based evaluation metric that determines the time taken by the whole formation to pass an obstacle and return back to the initial formation using the following:

$$
\mathrm{GSQ}=\mathrm{FQ}_{\text {Obstacle }}+\mathrm{FQ}_{\text {initial }}+\mathrm{FQ}_{\text {final }} \text {, }
$$

where $\mathrm{FQ}_{\mathrm{Obstacle}}$ is the time taken by the formation to pass the obstacle.
(3) Optimal formation divergence (OFD) is a metric used to determine the divergence of the built formation from the desired one and it sets the acceptability of this built formation with respect to a predefined tolerance which is defined by the task that this MRS will perform. OFD is a position-based evaluation metric that is calculated by the following:

$$
\mathrm{OFD}=\sum_{i=1}^{n} \frac{\mathrm{OAD}_{i}}{n}
$$

5.2. Evaluation Scenarios. Since there are two desired formations under investigation, it is required to have three evaluation scenarios for each formation. For each scenario, the evaluation metrics defined in the previous subsection 


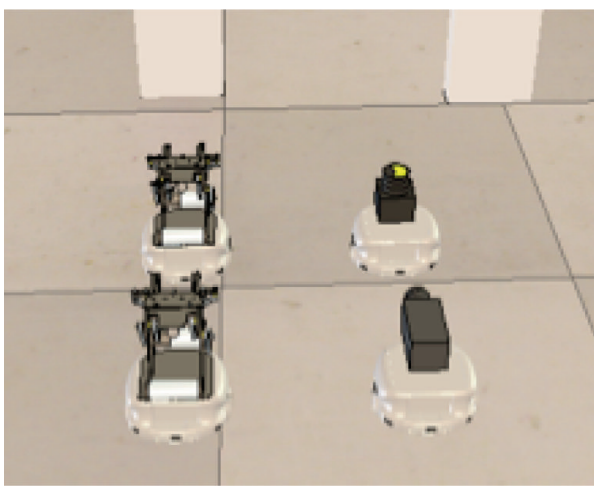

(a) Initial position

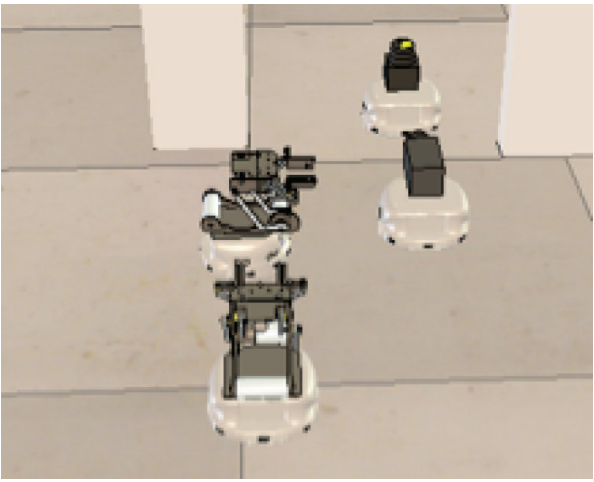

(c) State 1 achieved

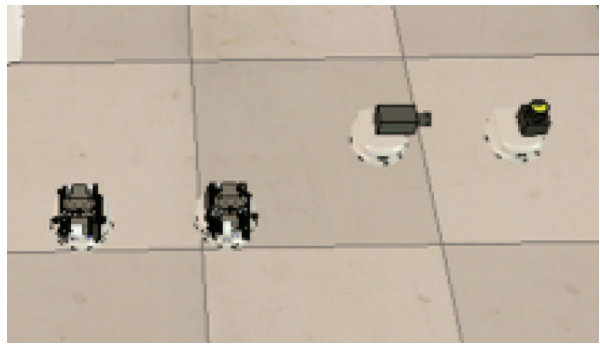

(e) Switching formation

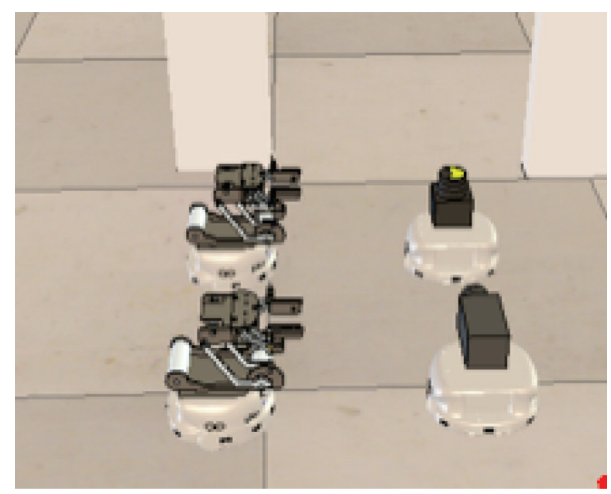

(b) Orienting

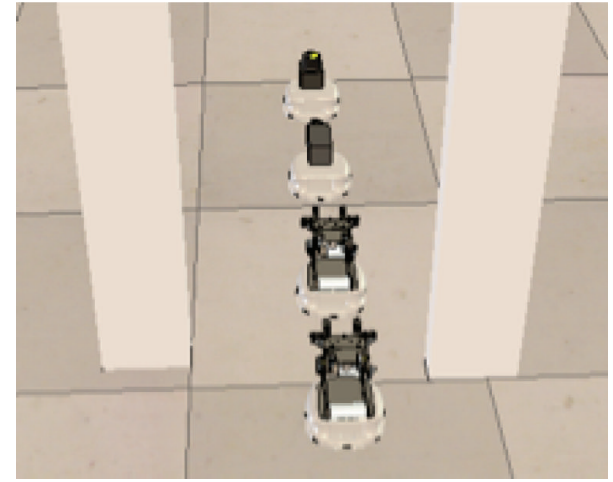

(d) Line formation

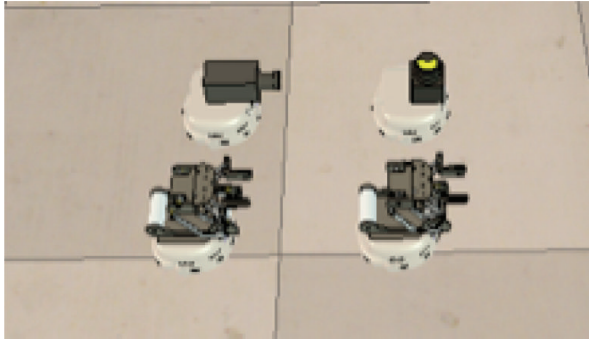

(f) Final position

FIGURE 23: Switching formation scenario.

will be used to evaluate the validity and efficiency of the approach used. The first evaluation scenario would be the ability of the approach under investigation to build up line formation (Figure 18) using randomly positioned agents in the environment (Figure 16).

The second scenario would be placing the agents in a random formation and setting a goal position to the MRS and investigating the ability of the algorithm to build a rectangle formation (Figure 19) and maintain the desired formation while in motion until reaching the goal position.

The third scenario investigates the adaptability of the algorithm where the environment is altered to have obstacles that forces the MRS to shift formation in order to pass the obstacle ahead (Figure 20).

(1) Line Formation (LF) Scenario. It is also known as column formation is built up using the 4 agents (robots). Initial position, the time taken to reach each state, the total state time (TST), and final position of each agent were reported. The following snapshots (Figure 21) were taken from the simulation platform showing the basic steps taken to change the MRS from a hazy formation into a line formation using the proposed approach.

(2) Rectangle Formation (RF) Scenario. It is basically two-line formations bonded by the virtual grid and communicating with each other. This formation is built up using the 4 agents (robots). Initial position, the time taken to reach each state, the TST, and final position of each agent were reported. The following snapshots (Figure 22) were taken from the simulation platform showing the basic steps taken to change the MRS from a hazy formation into a rectangle formation using the proposed approach.

(3) Adaptive Switching Formation Scenario. This scenario defines a rectangle formation that is desired to go through 


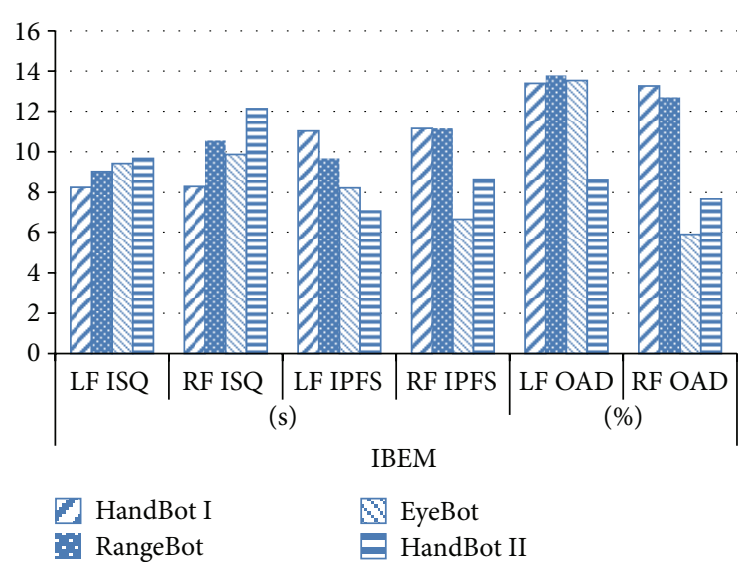

FIGURE 24: Individual behavioral EM.

a narrow passage; therefore, it switches the formation to line formation until passing the passage and then switches back to the rectangle formation. The 4 agents (robots) are introduced to this scenario in the rectangle formation and a narrow passage is set ahead of the formation. The following snapshots (Figure 23) were taken from the simulation platform showing the basic steps for switching the formation and then switching back to the original formation.

5.3. Evaluation Results. Figure 24 summarizes the IBEM for each agent in the MRS of the line formation and the rectangle formation scenarios. The chart shows that the average ISQ of the line formation is less than the ISQ of the rectangle formation and this is mainly because the line formation is easier to form than the rectangle formation. The IPFS of the line formation shows that each agent depends on the ahead agent and so they sequentially reach the desired positions, while the IPFS for the rectangle formation shows that each two agents are sequentially dependent on each other. Lastly, the $\mathrm{OAD}$ of the line formation shows larger divergence from the desired position than the rectangle formation and this is due to the small computational time taken by the line formation. These results conclude that the more the time taken to compute positions is, the less the divergence percentage is.

Figure 25 shows the collective behavioral EM for the line formation, rectangle formation, and the adaptation. The time taken to build the line formation is less than the time needed to build the rectangle formation while for the adaptation the FQ shows that agents in this MRS are able to quickly change formation from rectangle to line. The GSQ is only defined for the adaptation scenario as it is the total time taken for adaptation and the rebuilding of the initial formation. OFD shows that the adaptation scenario has the least divergence this is because of the large time taken for the computation of the final position.

\section{Conclusion}

This paper presented a hybrid approach to group formation problem in MRS. The proposed approach is based on dynamic leadership, cellular automata, and virtual grid. The

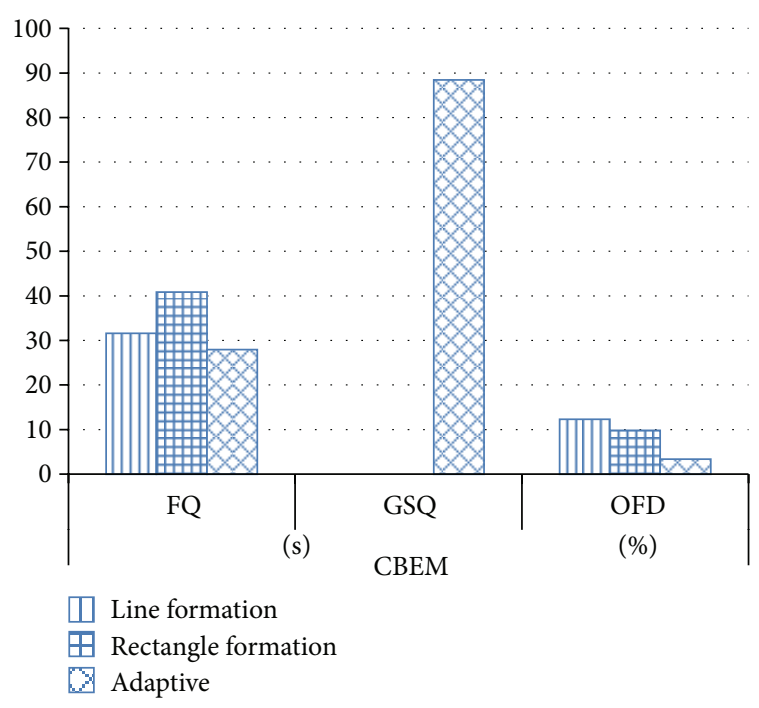

FIgURE 25: Collective behavioral EM.

cellular automata have an advantage of ensuring that the desired goal is achieved in form of milestones (checkpoints) and so provides the system with prompt feedback about the formation status, while the virtual grid provides the agents in the system with an interconnection technique that bonds the agents together and controls their behavioral activity. In this hybrid approach the rigidity of the automata theory is balanced by combining the allowance (tolerance) of the virtual grid technique with it.

The proposed approach has been proven by the experimental work that it can solve the problem of group formation in MRS. Also, the developed approach targeted the adaptability of the formation and how robust the agents in the MRS would react when the environment is changing. Results confirmed the hypothesis that claimed that the proposed hybrid approach is a sum of the advantages and suppressant of the disadvantages of its ingredients. Adaptability of the formation was tested using 4 agents switching from one formation to another due to the presence of obstacles in the environment. Moreover, the conducted experiments were set up using heterogeneous robots and results showed that the hybrid approach is fit for solving the group formation problem. However, more experiments are needed to verify the effect of communication failure on the stability of the proposed approach, also it is required to build more formations using this approach and qualitatively and quantitatively evaluate the performance. The proposed approach will be tested using Khepera III real robots in a newly built tested arena for MRS simulations and experiments in the robotics and autonomous systems (RAS) laboratory.

\section{References}

[1] R. C. Arkin, "Integrating behavioral, perceptual, and world knowledge in reactive navigation," Robotics and Autonomous Systems, vol. 6, no. 1-2, pp. 105-122, 1990.

[2] L. E. Parker, "Current research in multirobot systems," Artif Life Robotics, no. 7, pp. 1-5, 2003. 
[3] Y. Meng, J. Nickerson, and J. Gan, "Multi-robot aggregation strategies with limited communication," in Proceedings of the IEEE/RSJ International Conference on Intelligent Robots and Systems (IROS '06), pp. 2691-2696, October 2006.

[4] I. Mas and C. Kitts, "Object manipulation using cooperative mobile multi-robot systems," in Proceedings of the World Congress on Engineering and Computer Science (WCECS '12), San Francisco, Calif, USA, 2012.

[5] L. E. Parker, Path Planning and Motion Coordination in Multiple Mobile Robot Teams, Encyclopedia of Complexity and System Science, Springer, 2009.

[6] M. Luck, V. Marik, O. Štrpánková, and R. Trappl, Multi-Agent Systems and Applications, Springer, Berlin, Germany, 2001.

[7] B. J. Nelson, "Microrobotics in medicine. white paper," 2013, http://citeseerx.ist.psu.edu/viewdoc/download?doi= 10.1.1.98.994\&rep $=$ rep1\&type $=$ pdf.

[8] J. Leitner, "Multi-robot cooperation in space: a survey," in Proceedings of the Advanced Technologies for Enhanced Quality of Life (AT-EQUAL '09), pp. 144-151, July 2009.

[9] B. Nelson, I. Kaliakatsos, and J. Jake, "Microrobots for minimally invasive medicine," Annual Review of Biomedical Engineering, vol. 12, pp. 55-85, 2010.

[10] D. Davis and A. Pereira, Irregular Armed Forces and Their Role in Politics and State Formation, Cambridge University Press, 2008.

[11] K. A. Hawick, C. J. Scogings, and H. A. James, "Defensive spiral emergence in a predator-prey model," Tech. Rep., 2004.

[12] B. Lei, W. Li, and F. Zhang, "Flocking algorithm for multi-robots formation control with a target steering agent," in Proceedings of the IEEE International Conference on Systems, Man and Cybernetics (SMC '08), pp. 3536-3541, October 2008.

[13] Y. Q. Miao, A study of mobility models in mobile surveillance systems [M.S. thesis], Waterloo, Canada, 2010.

[14] K. Fujibayashi, S. Murata, K. Sugawara, and M. Yamamura, "Self-organizing formation algorithm for active elements," in Proceedings of the 21st IEEE Symposium on Reliable Distributed Systems (SRDS '02), pp. 416-421, October 2002.

[15] R. Daneshvar and L. Shih, Unifying Themes in Complex Systems VII, Springer, Berlin, Germany, 2012.

[16] Y. Cai and S. X. Yang, "A novel potential field-based pso approach to multi-robot cooperation for target searching in unknown environments," Seminar, 2012.

[17] J. Carroll and D. Long, Theory of Finite Automata: With an Introduction to Formal Languages, Prentice-Hall, 1989.

[18] 2013, http://www.coppeliarobotics.com/.

[19] N. Shrivastava, R. Mudumbai, U. Madhow, and S. Suri, "Target tracking with binary proximity sensors: fundamental limits, minimal descriptions, and algorithms," in Proceedings of the 4th International Conference on Embedded Networked Sensor Systems (SenSys '06), pp. 251-264, November 2006. 

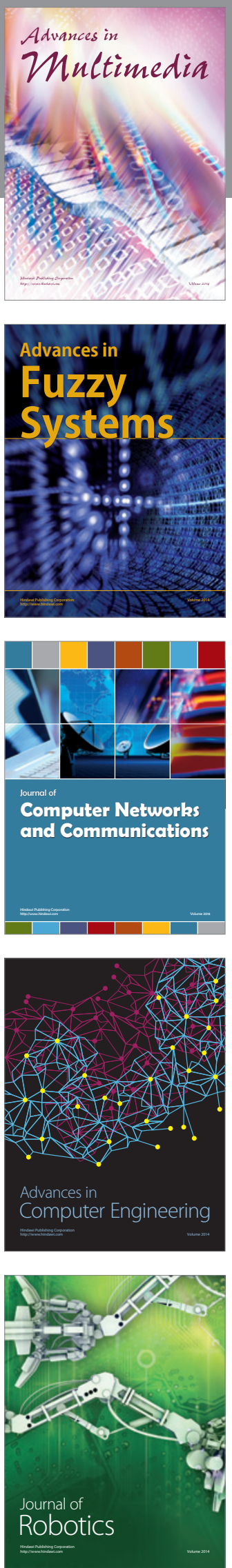

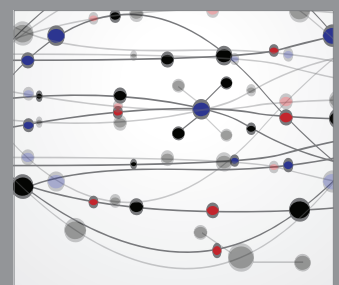

The Scientific World Journal
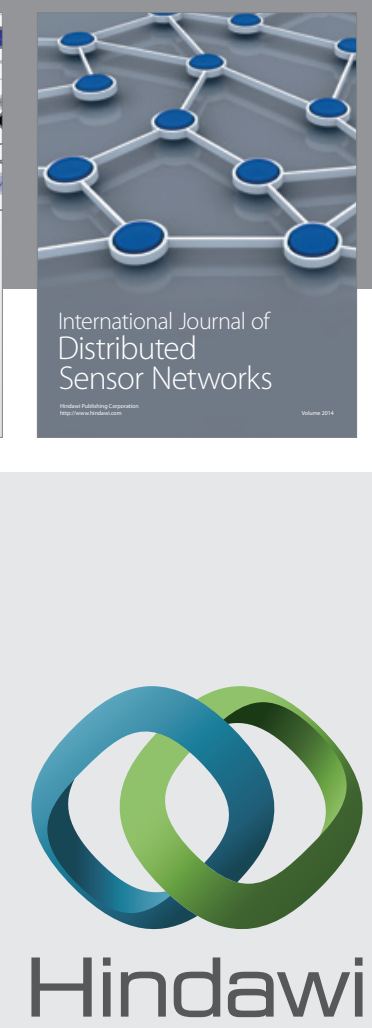

Submit your manuscripts at

http://www.hindawi.com
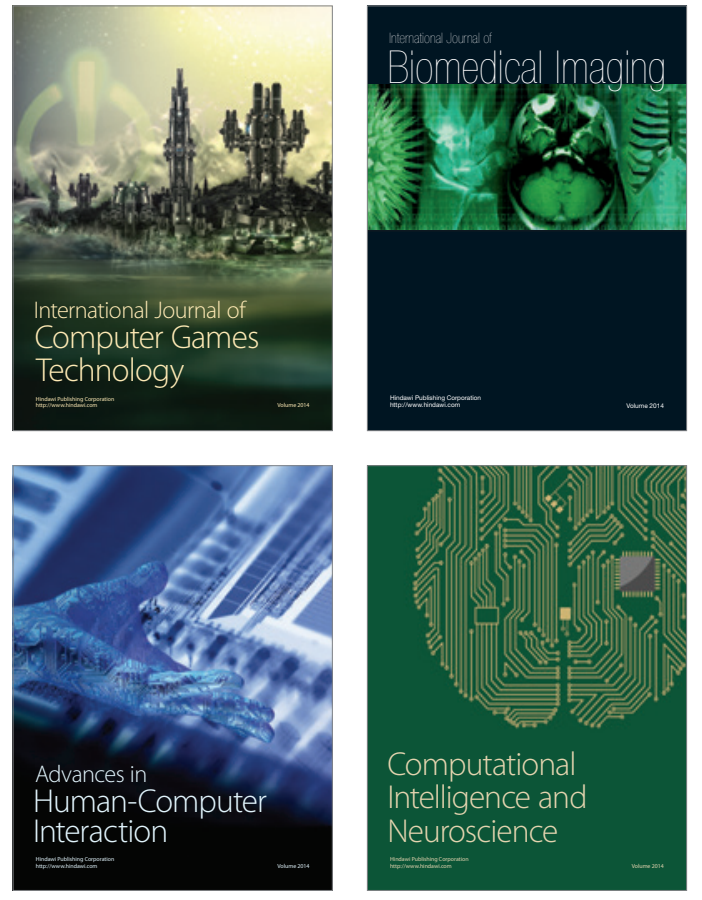
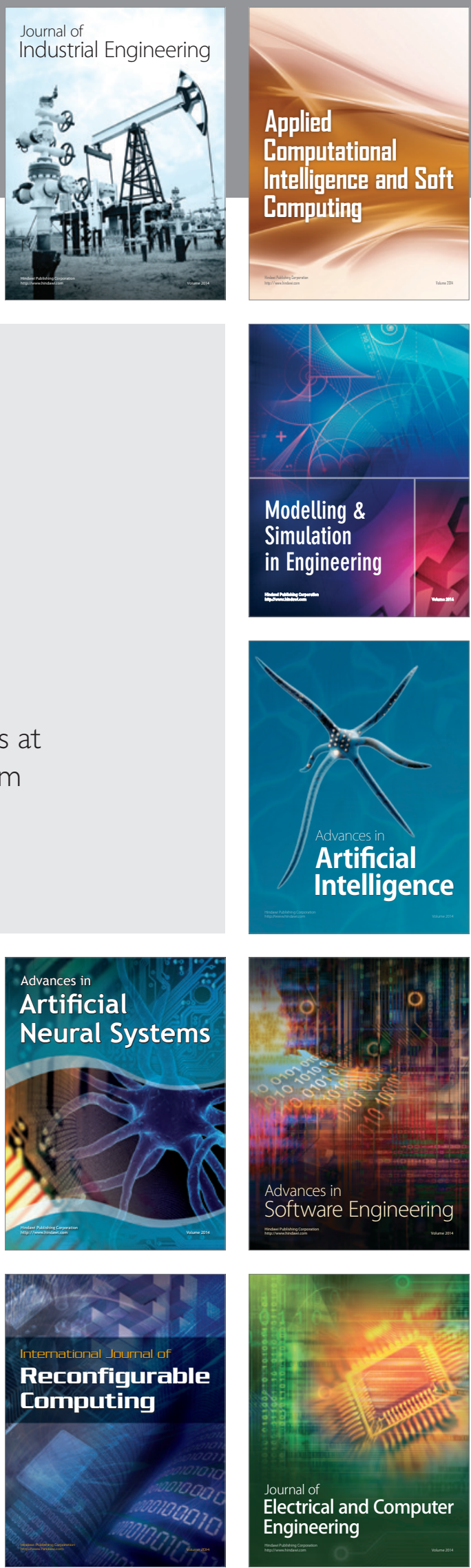